\title{
BinSanity: Unsupervised clustering of environmental microbial assemblies using coverage and affinity propagation
}

\author{
Elaina D Graham ${ }^{\text {Corresp.. }}{ }^{1}$, John F. Heidelberg ${ }^{1}$ ， Benjamin J. Tully ${ }^{1,2}$ \\ 1 Department of Biological Sciences, University of Southern California, Los Angeles, California, USA \\ 2 Center for Dark Energy Biosphere Investigations, Los Angeles, California, USA \\ Corresponding Author: Elaina D Graham \\ Email address: Egraham147@gmail.com
}

Metagenomics has become an integral part of defining microbial diversity in various environments. Many ecosystems have characteristically low biomass and few cultured representatives. Linking potential metabolisms to phylogeny in environmental microorganisms is important for interpreting microbial community functions and the impacts these communities have on geochemical cycles. However, with metagenomic studies there is the computational hurdle of 'binning' contigs into phylogenetically related units or putative genomes. Binning methods have been implemented with varying approaches such as k-means clustering, Gaussian mixture models, hierarchical clustering, neural networks, and two-way clustering; however, many of these suffer from biases against low coverage/abundance organisms and closely related taxa/strains. We are introducing a new binning method, BinSanity, that utilizes the clustering algorithm affinity propagation (AP), to cluster assemblies using coverage with compositional based refinement (tetranucleotide frequency and percent GC content) to optimize bins containing multiple source organisms. This separation of composition and coverage based clustering reduces bias for closely related taxa. BinSanity was developed and tested on artificial metagenomes varying in size and complexity. Results indicate that BinSanity has a higher precision, recall, and Adjusted Rand Index compared to five commonly implemented methods. When tested on a previously published environmental metagenome, BinSanity generated high completion and low redundancy bins corresponding with the published metagenome-assembled genomes. 
BinSanity: Unsupervised Clustering of Environmental Microbial Assemblies Using Coverage and Affinity Propagation

\author{
Elaina Graham ${ }^{1}$, John F. Heidelberg ${ }^{1}$, and Benjamin J. Tully ${ }^{2,1}$ \\ 1Department of Biological Sciences, University of Southern California, ${ }^{2}$ Center for Dark Energy \\ Biosphere Investigations
}

\title{
Abstract
}

Metagenomics has become an integral part of defining microbial diversity in various environments. Many ecosystems have characteristically low biomass and few cultured representatives. Linking potential metabolisms to phylogeny in environmental microorganisms is important for interpreting microbial community functions and the impacts these communities have on geochemical cycles. However, with metagenomic studies there is the computational hurdle of 'binning' contigs into phylogenetically related units or putative genomes. Binning methods have been implemented with varying approaches such as k-means clustering, Gaussian mixture models, hierarchical clustering, neural networks, and two-way clustering; however, many of these suffer from biases against low coverage/abundance organisms and closely related taxa/strains. We are introducing a new binning method, BinSanity, that utilizes the clustering algorithm affinity propagation (AP), to cluster assemblies using coverage with compositional based refinement (tetranucleotide frequency and percent GC content) to optimize bins containing multiple source organisms. This separation of composition and coverage based clustering reduces bias for closely related taxa. BinSanity was developed and tested on artificial metagenomes varying in size and complexity. Results indicate that BinSanity has a higher precision, recall, and Adjusted Rand Index compared to five commonly implemented methods. When tested on a previously published environmental metagenome, BinSanity generated high completion and low redundancy bins corresponding with the published metagenome-assembled genomes.

\section{Introduction}

Studies in microbial ecology commonly experience a bottleneck effect due to difficulties in microbial isolation and cultivation (J T Staley \& Konopka 1985). Due to the difficulty in culturing most organisms in a laboratory setting, alternative methods to analyze microbial diversity are commonly used to elucidate community structure and putative functionality. One such method is the sequencing of the collective genomes (metagenomics) of all microorganisms in an environment (Handelsman et al. 1998). Metagenomics can elucidate genomic potential, providing information on pathways, metabolism, and taxonomy allowing for inferences about environmental context without cultivation (Meyer et al. 2016). Grouping contigs into metagenome-assembled genomes (MAGs) is one of the hurdles faced when analyzing metagenomic data. Typically, one of a few issues are encountered in current binning protocols, including: decreasing accuracy for contigs below a size threshold, necessity of human intervention in distinguishing clusters, struggling to differentiate related microorganisms, or excluding low coverage and low abundance organisms (Alneberg et al. 2014; Bowers et al. 2015; Imelfort et al. 2014).

Popular unsupervised binning methods commonly use compositional parameters, such as tetranucleotide frequency (Anantharaman et al. 2016; Pride et al. 2003; Tully \& Heidelberg 2016; Tully et al. 2014), as the major delimiting parameter for creating putative groups of related sequences (bins). Due to the taxon specific nature of codon usage (Chen et al. 2004; Kanaya et al. 1999), GC content (Bohlin et al. 2010; Chen et al. 2004), and short oligonucleotides (k-mers) (Sandberg et al. 2001; Zhou et al. 2008), these fingerprints have been used to characterize and cluster contigs. However, the utilization of composition alone can lead to biases during the binning process for a number of reasons, including, closely related species having similar fingerprints and/or recently acquired genes from horizontal transfer, 
which can create chimeric bins that do not represent reality (Dick et al. 2009). Several methods and protocols have had increased success by incorporating coverage information as an additional variable during binning (Alneberg et al. 2014; Imelfort et al. 2014; Kang et al. 2015; Lu et al. 2016; Wu et al. 2014). Development of new binning protocols are essential for characterizing complex environmental communities and exploring microbial diversity at a level that cultivation-based studies presently cannot achieve.

BinSanity utilizes the clustering algorithm Affinity Propagation (AP) and accepts contig coverage values as the primary delimiting component. While other clustering algorithms can effectively group related DNA fragments using composition and coverage data, common methods, like hierarchical and kmeans clustering, require human input of information criteria that dictate the ultimate number of clusters (e.g., Bayesian information criterion). Assigning an a priori number for community diversity is increasingly difficult in complex ecosystems. AP, in contrast, requires no input on determining cluster centers; instead every point is iteratively considered as a potential cluster center. Data shows that AP effectively clusters a variety of data types and is often more precise than similar clustering methods (Chen-Chia et al. 2015; Flynn \& Moneypenny 2013; Frey \& Dueck 2007; Fujiwara et al. 2015; Gan \& Ng 2015; Hassanabadi et al. 2014; Leone et al. 2007; Walter et al. 2007; Zhengdong \& CarreiraPerpinan 2008). Though the implementation of AP for clustering contigs has been used before (Lin \& Liao, 2016), the primary method of clustering involved two composition based metrics, single copy marker genes and tetranucleotide frequencies. BinSanity, in contrast, bypasses possible composition based biases for binning contigs by creating an initial set of clusters determined using coverage. When necessary, these clusters can be refined with a composition based approach to deconvolute organisms with converging abundance values.

We benchmarked BinSanity by comparing it to five currently published binning software tools. We constructed several artificial microbial communities and created in silico metagenomic samples based on these sequences. The communities were composed of sequences that could be problematic for composition based binning algorithms, specifically metagenomes consisting of closely related and low abundance organisms. Additionally, a dataset associated with an infant gut microbiome time-series was used to establish how clusters generated via BinSanity compared to a highly curated set of genomic bins originally constructed using emergent self-organizing maps (ESOMs) (Dick et al. 2009). The results of this study find that BinSanity can generate high-quality genomes from metagenomics datasets via an automated process, which will enhance our ability to understand complex microbial communities.

\section{Methodology}

\section{Artificial Metagenomes}

In total, 60 reference genomes (including some closed genomes, some MAGs, and some draft genomes; Supplemental Table 1), consisting of a variety of organisms with ecological and environmental significance, were accessed from the Joint Genome Institute (JGI) Integrated Microbial Genome (IMG) Portal (Markowitz et al. 2014) and NCBI (Pruitt et al. 2007). These genomes were used to create in silico microbial communities. Reference genomes were screened via CheckM (Parks et al. 2015) to provide values of completion and contamination/redundancy based on single copy genes. Several combinations of the reference genomes were used to construct artificial communities (see below). For each community, in silico metagenomes were generated using the reads-for-assembly script (https://github.com/meren/readsfor-assembly), which generates "Illumina-like sequence reads" from the source DNA by mimicking random variations around an assigned coverage value and with simulated next-generation sequencing lengths and error rates. Because the script simulates variations around a mean-coverage value, genomes 
with assemblies greater than $20 \mathrm{kbp}$ (or closed genomes) were randomly split in to fragments between $3 \mathrm{kbp}$ and $15 \mathrm{kbp}$ in length using a Python script (split_file.py). For each community, 20 in silico metagenomes were created where each genome within the community had a different coverage value. In each iteration of a metagenome for an in silico community, organisms were assigned to be either low (randomly assigned a coverage value $<10 \mathrm{X}$ ) or high abundance (randomly assigned a coverage value between 10X-200X) by the script make_config_ini.py. The metagenomes were randomly selected to provide coverage values for the binning tools, with various tests performed on 2-20 in silico metagenomes.

Three artificial communities were constructed to test BinSanity and the other tools. The first artificial community selected 50 organisms from distinct species curated from the 60 reference genomes. Further referred to as diverse-mixture-1. In diverse-mixture-1, half of the organisms $(n=25)$ were randomly assigned to be either low or high abundance for each metagenomic sample. Organisms were independently assigned to the low and high abundance categories for each in silico sample. A second artificial community with 50 organisms was curated from the 60 reference genomes. This community, henceforth called diverse-mixture-2, assigned all organisms to be low abundance. The last artificial community contained 25 organisms, including four strains of Escherichia coli (further referenced as, strain-mixture). The strain-mixture randomly assigned organisms as low $(\mathrm{n}=13)$ or high abundance $(\mathrm{n}=$ 12).

After the reads for each in silico metagenome were generated, the reads were aligned back to the reference genomes using Bowtie2 (Langmead \& Salzberg 2012) (v2.2.5; default parameters). The output SAM file was then converted to a BAM file using SAMtools (Li et al. 2009) (v1.2 parameters: samtools view -bS file $\mid$ samtools sort - file). This BAM file was used to calculate the coverage for each contig (reads/bp) via an in-house script (contig-coverage-bam.py) that implements BEDtools (Quinlan \& Hall 2010). The determined coverage values were log transformed and results from multiple metagenomes were combined in to a single matrix using an in-house script (cov-combined.py).

Within BinSanity, each contig is evaluated as a possible exemplar based on the coverage. The exemplar is the contig that best represents the contigs clustering with it and can also be referred to as the cluster center. AP is described elsewhere (Flynn \& Moneypenny 2013; Frey \& Dueck 2007; Walter et al. 2007), but in brief, AP takes as input a collection of values where the similarity $\boldsymbol{s}(\boldsymbol{i}, \boldsymbol{k})$ indicates how well the data point with index $\boldsymbol{k}$ is suited to be the exemplar for data point $\boldsymbol{i}$. The messages sent between points make up either the responsibility $\boldsymbol{r}(\boldsymbol{i}, \boldsymbol{k})$ or the availability $\boldsymbol{a}(\boldsymbol{i}, \boldsymbol{k})$ (Frey \& Dueck 2007; Gan \& Ng 2015). The responsibility is the accumulated evidence that sample $\boldsymbol{k}$ should be the exemplar for sample $\boldsymbol{i}$ (Formula 1) (Walter et al. 2007). The availability (Walter et al. 2007) is the accumulated evidence that sample $\boldsymbol{i}$ should choose sample $\boldsymbol{k}$ to be its exemplar, dually considering the evidence of values for other samples that $\boldsymbol{k}$ should be an exemplar (Formula 2). Two limitations of AP are that it is hard to pinpoint the optimal preference (p) and damping factor. High values of a preference will lead to more exemplars (splitting) and low preferences will lead to a smaller number of exemplars (lumping). When setting a global value for AP, the minimum similarity is typically used as an initial choice for the preference (Frey $\&$ Dueck 2007). The damping factor is a number that helps to account for exemplars in periodic variance during the iterative process as well as improves convergence during oscillations (Mehmood \& Bie 2015; Zhengdong \& Carreira-Perpinan 2008). In addition, AP faces the challenge of time and memory complexity in the order of $\boldsymbol{O}\left(\boldsymbol{N}^{2} \boldsymbol{T}\right)$ where $\mathrm{N}$ is the number of samples and $\mathrm{T}$ is this number of iterations until convergence (Flynn \& Moneypenny 2013; Frey \& Dueck 2007; Mehmood \& Bie 2015; Walter et al. 2007). This order does not scale for production of a dense similarity matrix. 


$$
r(i, k) \leftarrow s(i, k)-\max _{k^{i} \text { s.t. } k^{i} \neq k}\left\{a\left(i, k^{\prime}\right)+s\left(i, k^{\prime}\right)\right\}(1)
$$

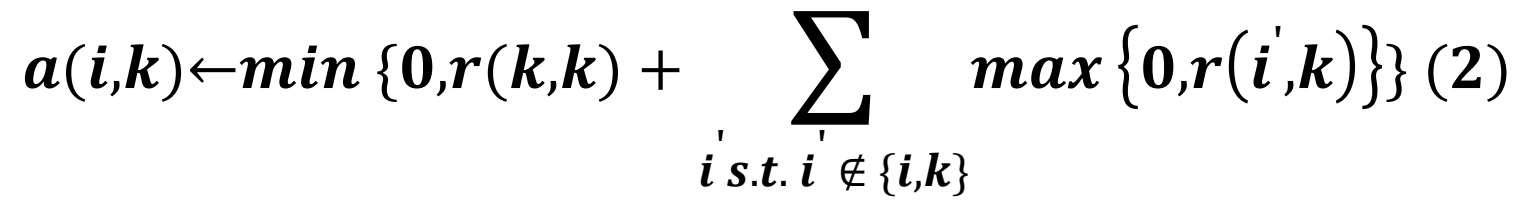 \\ $r(i, k) \leftarrow s(i, k)-\max _{a}\left\{a\left(i, k^{\prime}\right)+s\left(i, k^{\prime}\right)\right\}(1)$ \\ s.t. $i \notin\{i, k\}$}

BinSanity consists of two scripts, Binsanity.py and Binsanity-refine.py. BinSanity does an initial clustering of contigs based on the log transformed coverage as produced by contig-coverage-bam.py. First, a Euclidean distance similarity matrix is computed using scikit-learn. This matrix is used as input for AP (accessed via scikit-learn). The resultant cluster assignments for each contig are then used to generate FASTA files of each set of sequences. Several of the default settings can be modified depending on the nature of the metagenomic samples. Preference (-p) is used to adjust the degree to which AP will group or split contigs with similar coverages. A higher value will lead to a more stringent similarity requirement (i.e., create more clusters), whereas a smaller value will lead to more relaxed similarity requirements (i.e., create less clusters). Testing has shown that a preference value of $-10(-p-10)$ is successful, if used in conjunction with the refinement script (see below). Maximum iterations (-m) is the total number of iterations performed during clustering, if AP does not identify stable boundaries between clusters. If cluster boundaries are stable for the value given by the convergence iteration parameter (-v), then AP will stop before reaching the maximum iterations. Damping factor (-d) helps to account for contigs oscillating between two cluster centers across multiple iterations. Decreasing the damping factor could lead to uncontrolled oscillation that prevent AP from finding the optimal answer after the maximum iterations is reached.

BinSanity-refine.py is intended to be used following BinSanity.py and incorporates percent GC $(\% \mathrm{G}+\mathrm{C})$ and tetranucleotide frequencies to re-cluster contigs from high contamination and low completion bins. Convergence iteration, maximum iteration, contig cut off length, and dampening factor parameters are identical to the initial script. The default preference is decreased in this script (-p -150) to account for the extra input data provided by the $\% \mathrm{G}+\mathrm{C}$ and tetranucleotide frequencies. BinSanityrefine.py calculates both $\% \mathrm{G}+\mathrm{C}$ and tetranucleotide frequencies of the provided contigs. The script then proceeds as above.

BinSanity was executed on the log transformed coverage matrix using the script BinSanity.py (-m 4000 -v 400 -d 0.95 -x 1000 -p -10). BinSanity was compared against CONCOCT (Alneberg et al. 2014) (v.0.4.1; default parameters), GroopM (Imelfort et al. 2014) (v0.3.5; default parameters), MetaBat (Kang et al. 2015) (v0.26.3; default parameters), MaxBin (Wu et al. 2014) (v2.1.1; default parameters), and MyCC (Lin \& Liao 2016). All the methods were used with coverage information, if applicable.

BinSanity was tested with and without the refinement script. Initial analysis of the clustering results were conducted via CheckM (Parks et al. 2015) for completion, contamination, and strain heterogeneity. For this manuscript, 'contamination' values less than $10 \%$ will be referred to as 'redundancy', as multiple copies of a marker gene may not always represent 'contamination', but unmeasured diversity in the core genome of a phylogenetic group. After an initial round of binning based on coverage, bins determined by CheckM as highly contaminated or low completion, were subjected to a composition based refinement (BinSanity-refine.py; -m 4000 -v 400 -x 1000 -d 0.95 -p -150). Because reference organisms had known completion and low redundancy estimates, high completion bins were considered to be greater than $90 \%$ complete with less than $10 \%$ redundancy. Low completion bins were less than $90 \%$ complete and less 
172

173

174

175

176

177

178

179

180

181

182

183

184

185

186

187

188

189

190

191

192

193

194

195

196

197

198

199

200

201

202

203

204

205

206

207

208

209

210

211

than 5\% redundant. Any bins that did not fit in either low completion or high completion were labeled as high contamination. The Binsanity-refine.py script calculates $\% \mathrm{G}+\mathrm{C}$, tetranucleotide frequencies, and optionally will incorporate coverage to refine high contamination bins and re-cluster low completion bins. Tetranucleotide frequencies were scaled by 100 and $\log$ normalized. Results were evaluated by calculating precision, recall, and V-measure (e.g. harmonic mean) as defined by Rosenberg \& Hirschberg (2007) using sklearn.metrics.homogeneity_completeness_v_measure (Pedregosa et al. 2011) (bin_evaluation.py). Precision measured whether each cluster contains only members of a single class (output $=1$, all bins contain only contigs from a single source). Recall measured whether each member of a class is assigned to the same bin (output $=1$, only contigs from one source organism are contained in a single bin). The $\mathrm{V}$ measure was calculated as the harmonic mean of the precision and recall, allowing for the evaluation of accuracy. An additional measure, the Adjusted Rand Index (ARI) (Hubert \& Arabie 1985) was also calculated via sklearn.metrics.adjusted_rand_score (Pedregosa et al. 2011)

(bin_evaluation.py). The ARI considers similarity between predicted and true cluster labels by creating a contingency table comparing clusters. Within the context of this study, ARI analyzes the four possible situations that can arise when comparing determined cluster labels to the initial reference labels: (1) contigs are assigned to the same group in the reference and in the cluster; (2) contigs are in the same group in the reference and in different groups in the clusters; (3) contigs are in different groups in the reference and are assigned to the same group in the clusters; or, (4) contigs are in different groups in the reference and in different groups in the clusters. This similarity is then adjusted for chance using a probability heuristic. This adjustment accounts for the fact that given random cluster labeling you would expect to get a non-zero ARI. ARI analyzes the relation between elements in each class, in addition to these direct comparison of cluster labels (Santos \& Embrechts 2009). The general workflow for affinitypropagation is shown in Figure 1.

\section{Infant Gut Metagenome}

BinSanity was tested using samples from a time series study of an infant gut microbiome, previously described by Sharon et al. (2013). Samples were run though BinSanity.py (parameters: -p -10 m 4000 -v 400 -d 0.95 -x 1000). This same dataset was assessed by Eren et al. (2015) and was binned using a human guided strategy via the Anvi'o platform (Eren et al. 2015). In an effort to measure the effect of the binning algorithms (and to avoid influencing the results due to the use of different assemblers) the contigs produced by Eren et al. (2015) (http://anvio.org/data/) were used as the input for BinSanity (referred to as Eren-contigs). Raw reads were accessed from the NCBI SRA database (SRA052203) and aligned to the Eren-contigs. The coverage matrix was determined as described above. Additionally, the Eren-contigs were binned using CONCOCT, GroopM, MaxBin, MyCC and MetaBat. The Sharon et. al (2013) results were retrieved from All genome bins were evaluated via CheckM (Parks et al. 2015) and compared to genomes generated by Sharon et al. (2013) (http://ggkbase.berkeley.edu/carrol/). The Eren-contigs were Blast searched against the Sharon et al. (2013) contigs so that contig ids for each could be visually compared (results available http://merenlab.org/tutorials/infant-gut/). To maintain consistency, the curated bins from Sharon et al. (2013) were processed using CheckM, so that all single gene copy based redundancy and completion metrics were consistent. 


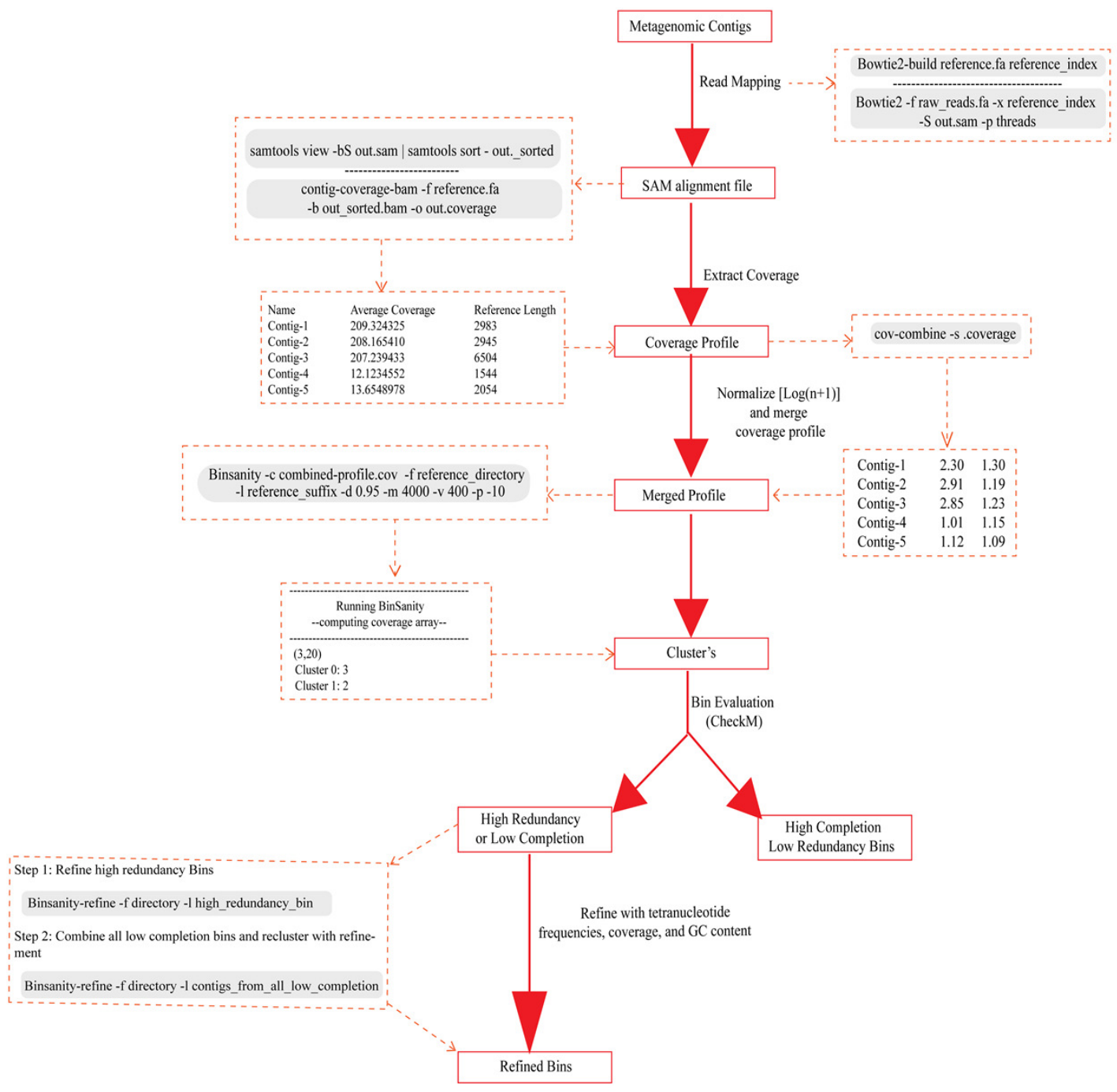

212

Figure 1. Workflow for Binsanity indicating all scripts used.

\section{Results and Discussion}

Species Level: Diverse-Mixture-1

In processing diverse-mixture-1, BinSanity+refinement had near perfect results with an ARI and V-measure of 0.98 using 20 in silico metagenomes (Figure 2). When the number of in silico metagenomes was decreased to five, BinSanity had the highest ARI at 0.97, while BinSanity+refinement had an ARI of 0.95. With this number of metagenomic samples, BinSanity produced the highest V-measure score of the binning methods, indicating it most closely reconstructed the reference organisms and had minimal rates of incorrectly assigning contigs. Without the use of refinement, BinSanity produced 54 bins of which one had high contamination $(>10 \%)$, and five were low completion ( $<85 \%$ complete). When BinSanity+refinement was implemented 52 bins were produced (Table 1). Of those 52 bins, two were less than $20 \%$ complete and contained contigs originating from a single reference organism. 
A

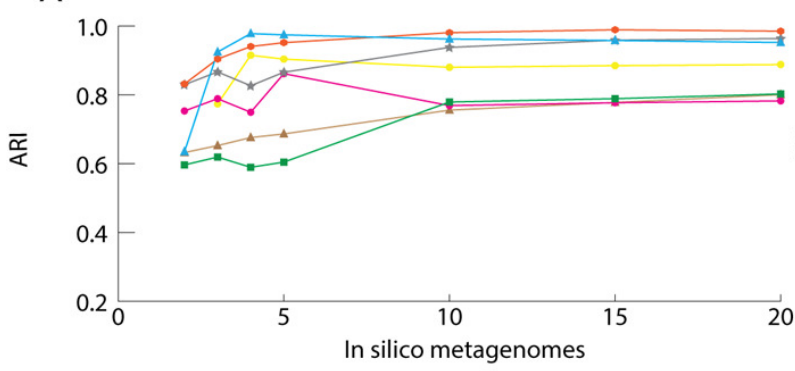

C

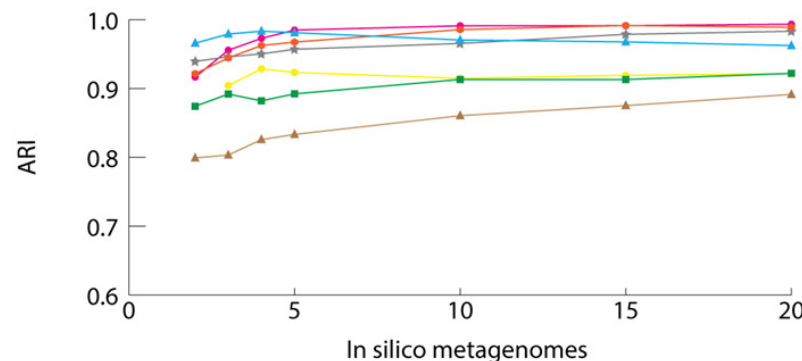

B

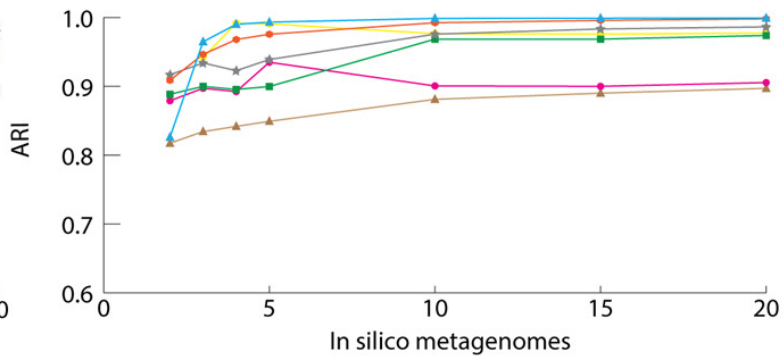

D

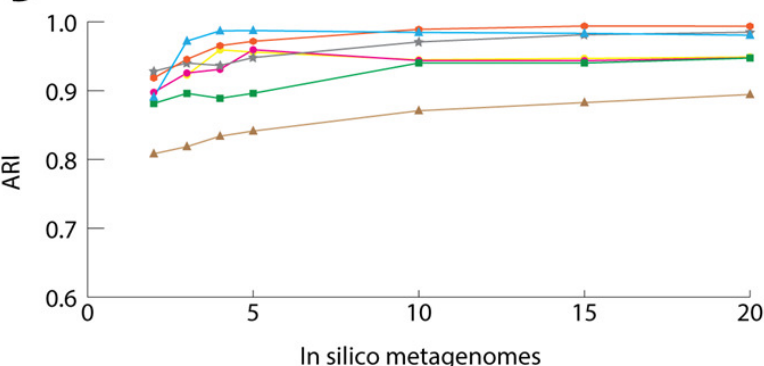

$\leadsto$ BinSanity $\rightarrow$ BinSanity+refinement $\rightarrow$ CONCOCT $\rightarrow$ MetaBat $\rightarrow$ MaxBin $\rightarrow$ GroopM $\rightarrow$ MyCC

Figure 2. Stastistical calculations (bin_evaluation.py) showing the adjusted rand index (ARI) (A), precision (B), recall (C), and V-measure (D) for diverse-mixture-1.

229 In comparison to BinSanity, CONCOCT, GroopM, MyCC and Metabat had high precision and low recall, 230 producing more bins than expected (71-109 bins), while MaxBin had high recall and low precision producing less bins (42), when using coverage data from five in silico metagenomes (Table 1).

\begin{tabular}{|c|c|c|c|c|c|c|c|c|}
\hline \multicolumn{7}{|c|}{ Table 1. Number of Bins Produced by Each Method for each number of in silico metagenomes } \\
\hline \multicolumn{7}{|c|}{ Diverse-mixture-1 $(\mathrm{n}=50)$} \\
\hline In silico metagenome & BinSanity & BinSanity+refinement & CONCOCT & GroopM & MetaBat & MaxBin & MyCC \\
\hline 2 & 32 & 46 & 70 & -- & 73 & 44 & 107 \\
\hline 3 & 38 & 51 & 64 & 102 & 74 & 41 & 110 \\
\hline 4 & 52 & 51 & 68 & 109 & 74 & 39 & 106 \\
\hline 5 & 54 & 52 & 71 & 109 & 71 & 42 & 103 \\
\hline 10 & 64 & 53 & 73 & 86 & 81 & 38 & 99 \\
\hline 15 & 51 & 52 & 71 & 87 & 31 & 37 & 98 \\
\hline 20 & 72 & 55 & 69 & 81 & 78 & 38 & 83 \\
\hline
\end{tabular}




\begin{tabular}{|c|c|c|c|c|c|c|c|}
\hline 3 & 33 & 50 & 70 & 59 & 73 & 44 & 127 \\
\hline 4 & 41 & 50 & 72 & 58 & 71 & 43 & 124 \\
\hline 5 & 46 & 50 & 71 & 92 & 69 & 41 & 123 \\
\hline 10 & 52 & 50 & 62 & 68 & 73 & 43 & 126 \\
\hline 15 & 54 & 51 & 57 & 78 & 74 & 40 & 144 \\
\hline 20 & 55 & 51 & 55 & 60 & 75 & 37 & 160 \\
\hline \multicolumn{8}{|c|}{ Strain mixture $(n=25)$} \\
\hline in silico metagenome & BinSanity & BinSanity+refinement & CONCOCT & GroopM & MetaBat & MaxBin & MyCC \\
\hline 2 & 21 & 17 & 33 & -- & 38 & 25 & 85 \\
\hline 3 & 23 & 22 & 34 & 34 & 53 & 18 & 53 \\
\hline 4 & 28 & 25 & 35 & 50 & 46 & 19 & 53 \\
\hline 5 & 30 & 25 & 34 & 63 & 48 & 18 & 55 \\
\hline 10 & 35 & 26 & 32 & 41 & 45 & 22 & 63 \\
\hline 15 & 39 & 26 & 28 & 58 & 47 & 21 & 57 \\
\hline 20 & 42 & 27 & 25 & 41 & 42 & 18 & 46 \\
\hline
\end{tabular}

232

Of the 47 bins produced by MaxBin, eight were highly chimeric (Figure 3). When isolating the bins that contained $>90 \%$ complete genomes, BinSanity produced 46 bins, while MetaBat and GroopM produced 33 and 41, respectively. CONCOCT, overall, had a high accuracy, but had difficulty delimiting closely related species such as Roseobacter denitrificans and $R$. litoralis. This difficulty in separating closely related species could be related to the use of a single step clustering protocol, where composition and coverage are used as equally weighted inputs. Closely related organisms often have similar composition signals, while coverage is reliant on the underlying population of the organisms in the community. This can lead to instances where contigs from similar strains cannot be teased apart using compositional data, but can be separated based on coverage values over multiple samples.

It can be difficult to distinguish strains using coverage based methods if reads are not stringently assigned due to bias within conserved regions and nonspecific alignment. Strict alignment parameters (such as using the --very-sensitive flag in Bowtie2) can be used to prevent false contig assignments and increase fidelity of all the binning methods. Additionally, more coverage information, especially variable coverage data, benefits all the methods, as is evident when analyzing results generated using $<5$ in silico metagenomic samples; all methods decline in accuracy (Figure 2). 


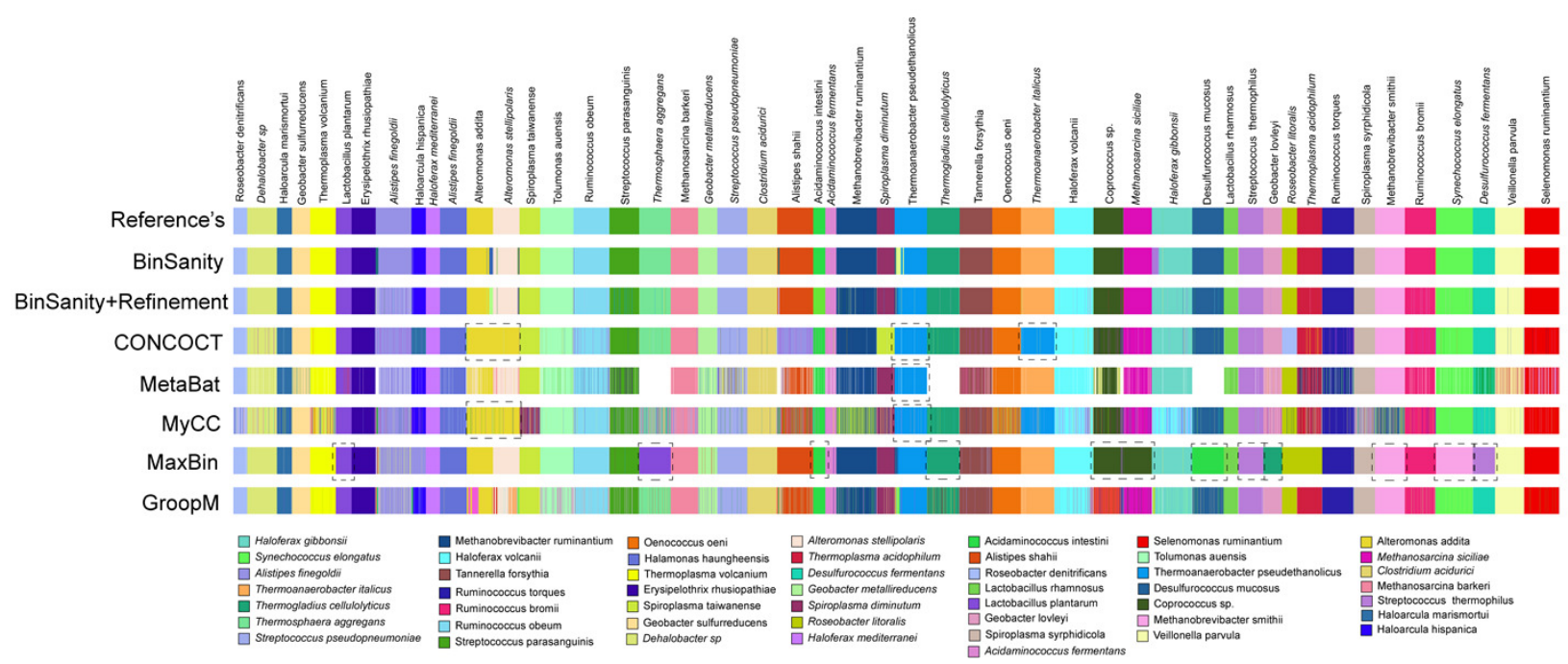

Figure 3. Clustering results for diverse-mixture-1 BinSanity, BinSanity+refinement, CONCOCT, MetaBat, MyCC, MaxBin, and GroopM at five in silico metagenomes (visualized via Anvi'o). Black dashed boxes highlight bins in each method that contained contigs from two or more reference organisms. White represents those contigs that were left un-clustered.

The primary method for generating bins within BinSanity is clustering using coverage values. When the number of in silico metagenomes decreases (for example, $<5$ metagenomes), there is an insufficient amount of information to differentiate between low coverage organisms with similar abundances across multiple samples. At four in silico metagenomes, BinSanity grouped organisms with similar coverage profiles together, leading to some bins with high contamination. Utilizing the refinement method to differentiate bins with high contamination increased the precision and recall values when the amount of coverage data was limited. When using refinement with data from two in silico metagenomes, BinSanity returned the highest ARI value (Figure 2).

Species Level: diverse-mixture-2

In diverse-mixture- 2 (all organisms $<10 \mathrm{X}$ coverage), the initial clustering step from BinSanity decreased in accuracy (e.g., decreased ARI, precision, and V-measure) when using data from $<10$ in silico metagenomes, though maintained near perfect ARI scores when $\geq 10$ samples were tested (Figure 4). This trend was expected, as the convergence of coverage from multiple organisms would lead to contigs from multiple taxa being clustered into the same bin. Utilization of the refinement method, resolved many of these artificial clusters (Supplemental Figure 1), such that BinSanity+refinement achieved ARI scores of 0.99 when $\geq 10$ samples were used for clustering and maintained the highest ARI value when 3-5 in silico metagenomes were used; at two to three metagenomic samples, BinSanity was outperformed by CONCOCT. 
A

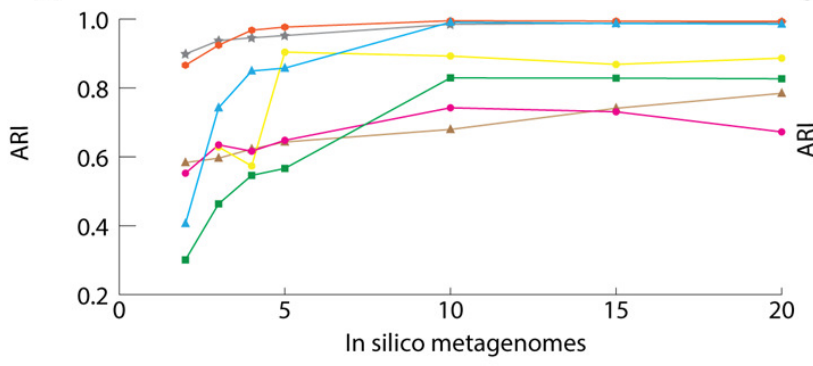

B

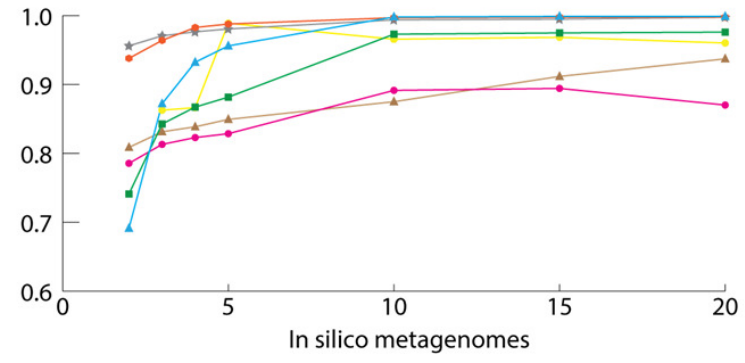

C

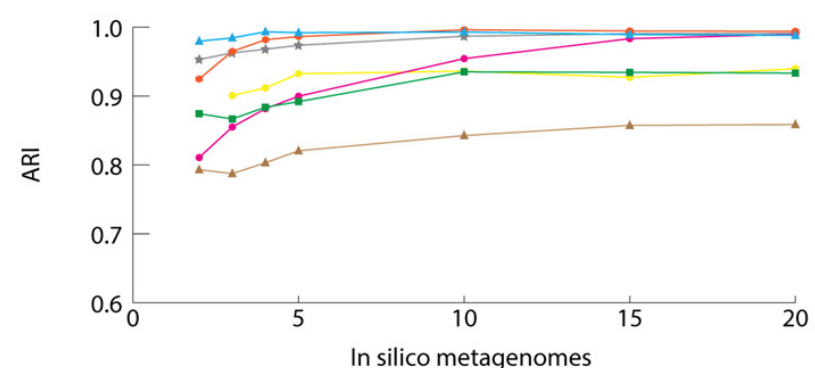

D

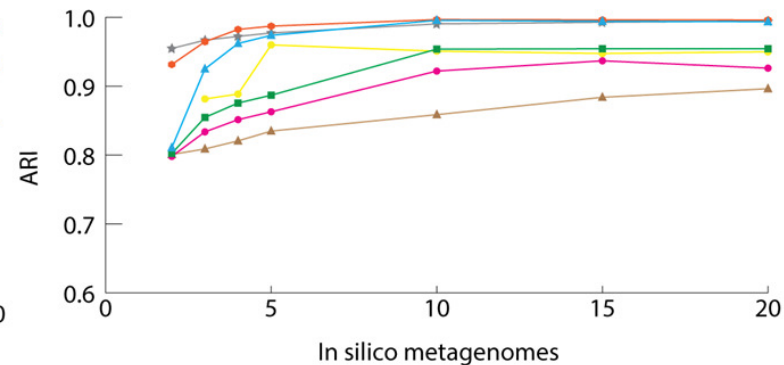

$\rightarrow$ BinSanity $\rightarrow$ BinSanity+refinement $\rightarrow$ CONCOCT $\rightarrow$ MetaBat $\rightarrow$ MaxBin $\rightarrow$ GroopM $\leadsto$ MyCC

271

272

273

274

275

276

277

278

279

280

281

282

283

284

285

286

287

288

289

290

291

292

Figure 4. Statistical calculations (bin_evaluation.py) showing Adjusted Rand Index (A), Precision (B), Recall (C), and V-Measure (D) for diverse-mixture-2.

Comparison of CONCOCT, MaxBin, MetaBat, GroopM, BinSanity, and BinSanity+refinement at five in silico metagenomes, indicated that BinSanity+refinement produced bins with a higher degree of agreement to the true contig assignments (Figure 4). At five in silico metagenomes, BinSanity (without refinement) produced 46 bins compared to an input of 50 genomes. When refinement was incorporated into the workflow, BinSanity+refinement was able to resolve the 50 bins. BinSanity+refinement could accurately split contigs from six organisms that were clustered into two bins during the initial BinSanity step. As with the previous community test, CONCOCT, GroopM, MyCC and MetaBat produced more than the input genomes (69-123 bins), while MaxBin created 41 bins. CONCOCT and GroopM produced results with more accuracy than MaxBin, MyCC, and MetaBat. However, GroopM failed to cluster one organism and over split several other organisms, and CONCOCT clustered two Desulfurococcus species and over split several genomes. MetaBat massively over split genomes and had a high percentage of contigs $(14.84 \%)$ that were not placed in bins. MyCC, similar to MetaBat, over split multiple genomes, but had lower instances of bins containing multiple taxa. These results suggest BinSanity can separate low coverage organisms effectively from a large sample set by conducting a first pass using the standard BinSanity script, followed by refinement of bins with high contamination and/or low completion.

\section{Strain-Level}

For the strain-mixture community with 25 organisms (including 4 strains of Escherichia coli), BinSanity produced 30 bins when using data from five metagenomes. When refinement was used to re- 
302

cluster high contamination and low completion bins, the output was reduced to the target 25 bins. In contrast, CONCOCT, MetaBat, MyCC, and GroopM over split the data (34-63 bins), while MaxBin did not generate the input number of genomes (19 bins). These tools all had lower overall values for the other determined metrics compared to BinSanity+refinement (Figure 5). BinSanity maintained the highest ARI and $\mathrm{V}$-measure regardless of the number of metagenomes used to generate the coverage values. While GroopM and MetaBat did created more bins than the number of target genomes, those bins did have high precision (i.e., a low percentage of bins contained contigs from multiple lineages). MyCC and CONCOCT had difficulty delineating some of the E. coli strains and the two Escherichia species (Supplemental Figure 2).

A

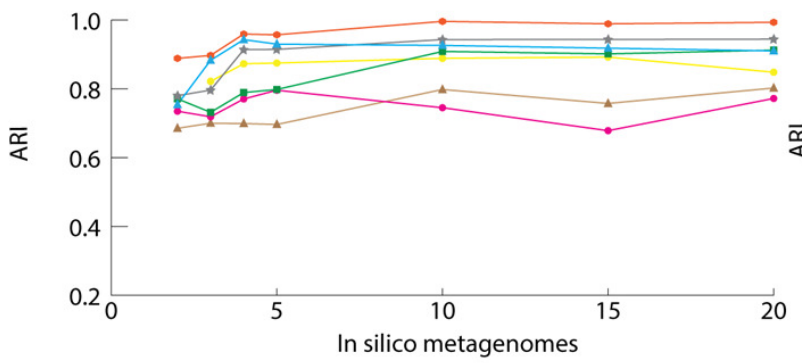

C

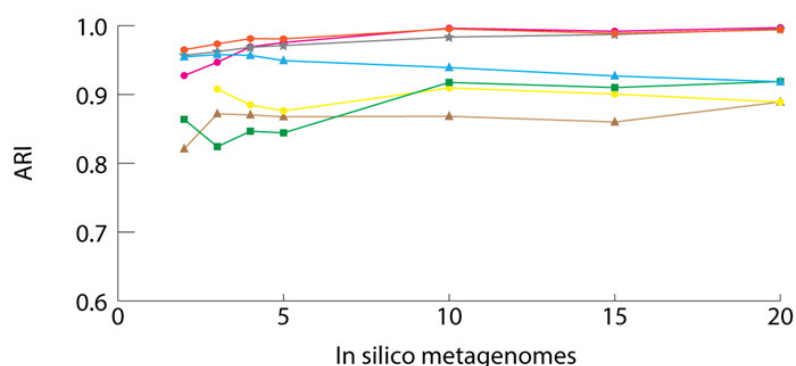

B

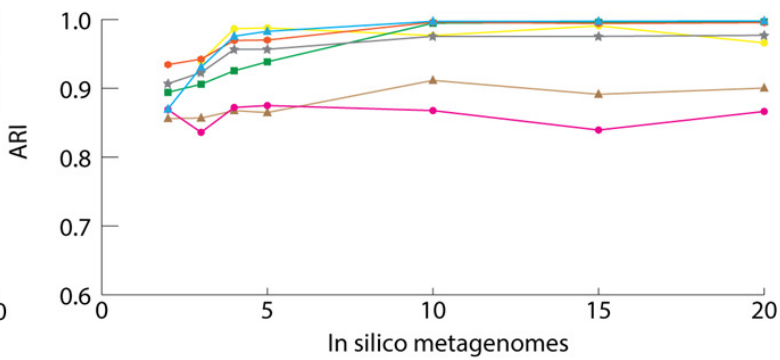

D

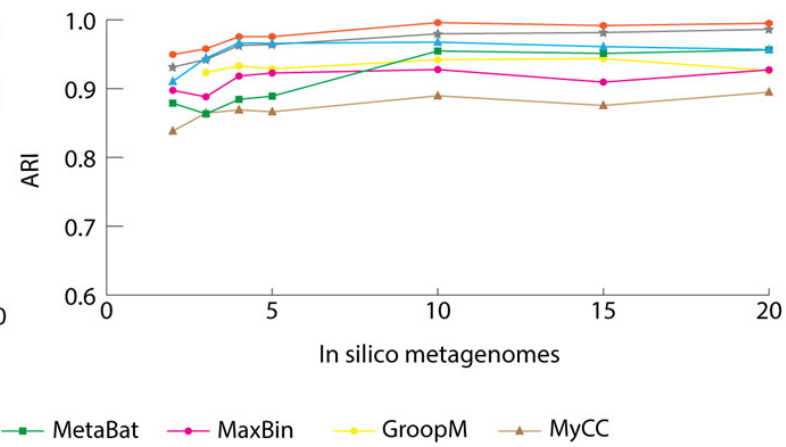

$\leadsto$ BinSanity $\rightarrow$ BinSanity+refinement $\rightarrow$ CONCOCT $\rightarrow$ MetaBat $\rightarrow$ MaxBin $\rightarrow$ GroopM $\rightarrow$ MyCC

Figure 5. Statistical calculations (bin_evaluation.py) for Adjusted Rand Index (A), Precision (B), Recall (C), and V-Measure (D) for the strain-mixture.

The primary difficulty for clustering this dataset for all the tested methods was accurately differentiating organisms with strain-level similarity. BinSanity+refinement produced 25 bins. Of the 25 bins produced 3 had high contamination and high strain heterogeneity. One of these bins was 91\% complete with $68.39 \%$ contamination and $99.50 \%$ strain heterogeneity (Supplemental Table 2). This bin contained contigs from Escherichia coli $083 H 1$ (4.1\%), E. coli UMNO26 (12.3\%), and E. coli 0104 H4 (81\%). The second bin was $84.64 \%$ complete with $13.79 \%$ contamination and $95.83 \%$ strain heterogeneity. This bin primarily contained contigs from E. coli $083 H 1(98 \%)$ but also contained contigs from E. fergusonii. The third bin was $68.97 \%$ complete with $8.62 \%$ contamination and $100 \%$ strain heterogeneity. This bin contained contigs from E. coli UMNO26 (70\%), E.coli $083 H 1(0.84 \%)$, and E. coli $0104 \mathrm{H} 4$ (28\%). E. coli $0104 \mathrm{H} 4$ and E. coli UMN026 were the least accurately clustered with contigs being placed into 2 and 4 bins respectively. MaxBin achieved the best resolution of the E. coli strains, but 
316

317

318

319

320

321

322

323

324

325

326

327

328

329

330

331

332

333

334

335

336

337

338

339

340

341

342

343

344

345

346

347

348

349

had difficulty clustering other organisms within the community (Supplemental Figure 2). Metabat and GroopM had high precision, but an extremely low recall due to high degree of genome splitting. CONCOCT, although approximating the correct results for the other members of the community, largely clustered all 6 Escherichia genomes into a single bin.

For the strain-mixture community, GroopM, MetaBat, and MaxBin failed to cluster the most contigs, 261, 56, and 49 contigs, respectively. BinSanity fared better than CONCOCT in accurately representing strains. Based on both the statistics (ARI, precision, and recall) and binning output analysis, BinSanity performed better than the current published unsupervised methods for clustering a community with strain-level variation.

\section{Infant Gut Metagenome}

BinSanity was applied to a metagenomic dataset from a time-series of samples collected from an infant gut environment by Sharon et al. (2013) and assembled by Eren et al. (2015). The CLC assembled contigs were processed using BinSanity, CONCOCT, GroopM, MaxBin, MyCC, and MetaBat (Figure 6). The results from the BinSanity method were additionally compared to the output generated by Sharon et al. (2013) and Eren et al. (2015) (Table 2). The Eren et al. (2015) bins were curated using human guided binning via Anvi'o, and the Sharon et al. (2013) genomes were generated via ESOM (Dick et al. 2009) and manual curation. Without refinement, BinSanity closely resembled the previously identified bins/genomes.

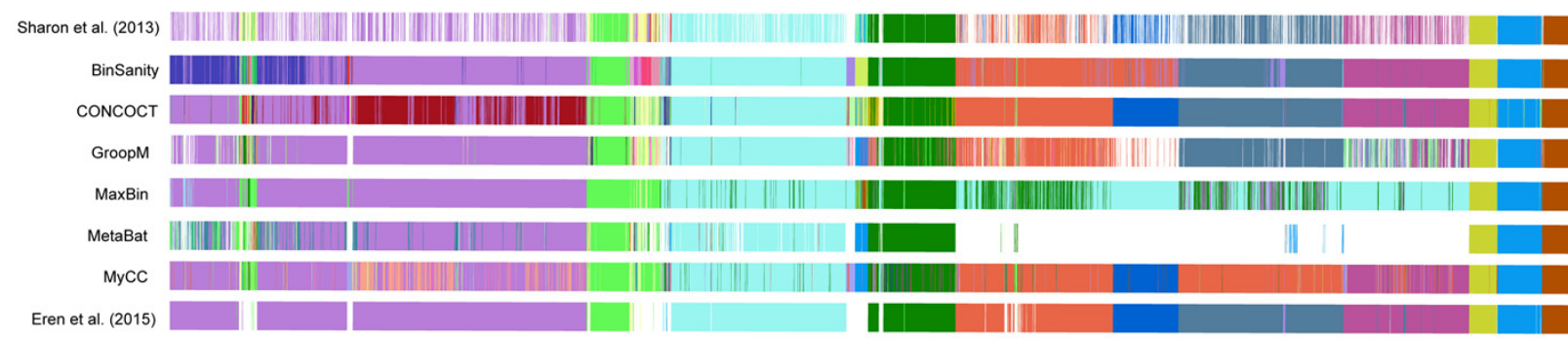

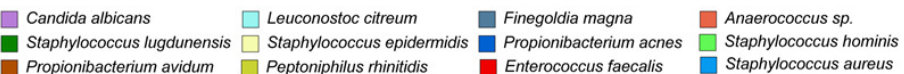

Sreptococcus pneumoniae - Propionibacterium avidum $\square$ Peptoniphilus rinitidis Enterococcus faecalis

Figure 6. Clustering of the infant gut metagenome by BinSanity, CONCOCT, GroopM, MaxBin, MetaBat, MyCC, Eren et al. (2015), and Sharon et al. (2013). The image was generated Anvi'o.

BinSanity had minor issues in resolving three of bins from the dataset. BinSanity split contigs assigned to Staphylococcus epidermidis into two bins, generating a bin with a majority of the $S$. epidermidis contigs that was $89.1 \%$ complete, in comparison to $90.3 \%$ and $99.8 \%$ complete genomes determined via Anvi'o and Sharon et. al., respectively. BinSanity without refinement clustered the genome fragments assigned by Sharon et. al. as Propionibacterium acnes (5.6\% complete) and Anaerococcus $(2.51 \%$ complete) into a single bin. These organisms were present at such low abundance, as revealed by their incomplete nature, that BinSanity could not resolve this delineation. When BinSanity+refinement was applied, the results mirrored that of Anvi'o, with a single Anaerococcus bin at $\sim 10 \%$ complete and $P$. ances bin at $0 \%$ complete (refined contig assignments provided in Supplemental Table 3). Candida albicans, a eukaryote, was difficult to cluster accurately for all three methods (Table 2). However, this can be expected as the task of accurately clustering DNA from eukaryotic genomes is currently beyond the scope of BinSanity and many of the methods discussed in this research. BinSanity was able to accurately cluster four bins that mapped back to the Staphylococcus aureus virus, 
350 351

352

Propionibacterium virus, S. epidermidis virus 013, and S. epidermidis virus 014 described by Sharon et al. (2013).

\begin{tabular}{|c|c|c|c|}
\hline Bin ID & ESOM (Sharon et al. 2013) & BinSanity & Anvi'o (Eren et al. 2015) \\
\hline Staphylococcus aureus_33_1 & $99.51(0.08)$ & $95.02(0.66)$ & $95.02(0.66)$ \\
\hline Staphylococcus lugdunensis_33_1 & $84.10(0.02)$ & $58.07(1.72)$ & $58.07(1.72)$ \\
\hline Staphylococcus epidermidis_32_1 & $99.81(0.00)$ & $89.06(0.00)$ & $90.28(2.22)$ \\
\hline Staphylococcus_hominis_M0480 & $95.39(0.57)$ & $97.26(2.42)$ & $97.73(2.19)$ \\
\hline Peptoniphilus harei_30_1 & $98.95(0.00)$ & $100(0.00)$ & $100(0.00)$ \\
\hline Propionibacterium_63_1 & $97.86(0.00)$ & $98.95(0.00)$ & $98.95(0.00)$ \\
\hline Enterococcus faecalis_37_1 & $99.25(0.00)$ & $99.63(0.00)$ & $99.53(0.00)$ \\
\hline Leuconostoc citreum_37_1 & $45.64(0.23)$ & $62.94(2.57)$ & $62.80(2.57)$ \\
\hline Candida albicans_32_1 & $34.43(9.48)$ & $60.89(26.92)$ & $61.76(27.65)$ \\
\hline Finegoldia magna_32_1 & $29.25(0.00)$ & $32.54(0.29)$ & $35.43(0.60)$ \\
\hline Streptococcus_mitis_38 & $16.45(0.33)$ & $25.31(1.00)$ & $23.10(0.25)$ \\
\hline Propionibacterium_acnes & $5.64(0.00)$ & $0(0.00)$ & $0.00(0.00)$ \\
\hline Anaerococcus_18_1 & $2.51(0.00)$ & $11.02(1.22)$ & $9.90(0.00)$ \\
\hline Archaea_unk & 0.00 & $6.00(0.00)$ & 0.00 \\
\hline
\end{tabular}

353

BinSanity closely approximated the manually derived Anvi'o results with higher accuracy than the other unsupervised methods. CONCOCT clustered Anaerococcus and Finegoldia magna, while creating two highly chimeric bins from four other organisms. MetaBat failed to cluster a significant majority of the contigs (69\%). MaxBin had difficulty identifying four organisms that were $<50 \%$ complete and had low contig coverage. GroopM resembled both the BinSanity and Anvi'o results, but overall the bins were less robust and contained less contigs. MyCC had difficulty distinguishing between F. magna and Anaerococcus sp.

Due to the use of the CLC assembled infant gut contigs generated by Eren et. al. (2015) and not the original contigs from the Sharon et. al. (2013) study (contigs are not publically available), some variation in the results the other methods are present. These variations can be seen in the Staphylococcus bins. For example, S. lugdunensis was determined to be $\sim 58 \%$ complete by BinSanity, Anvi'o, and CONCOCT (MetaBat at $49 \%$ complete), but the genome published by Sharon et. al. was $84 \%$ complete. Overall, BinSanity generated bins reflecting published organisms from this metagenome sampling.

\section{A Note on Assigning a Preference Value \& The Memory Complexity}


BinSanity is sensitive to changes in the preference value. The preference value sets limits as to how relaxed or stringent AP should be in deciding the number of cluster centers. Although we found high success using the provided default values for BinSanity, results can be optimized for different sample scenarios by taking in to account the complexity and coverage of the microbial community within a sample. When a high range of coverages exists, the preference can be reduced to prevent over splitting the assemblies. When a low range of coverages exists, the preference can be increased to prevent inaccurate clustering of contigs. If strain-level diversity is high, the preference can be inversely scaled to the number of metagenome replicates (e.g., the more metagenomic samples the lower the preference). Iteratively testing preferences is the best way to find the optimal clustering result while using BinSanity. Recommendations from the authors of AP suggest a good starting point for the preferences is the median or minimum similarity between the most extreme values (Frey \& Dueck 2007). We recommend using BinSanity, with preference values that favor higher recall (e.g., using a lower preference, such as the default value of -10) because the refinement script can the successfully separate organisms with similar coverage profiles.

Due to the implementation of AP, parallel computing options are not currently available. AP is a deterministic algorithm meaning, such that re-running the script on identical data will always yield the same clustering results. Clustering diverse-mixture-1 with 27,643 contigs on a Dell PowerEdge R920 with $1 \mathrm{~TB}$ of available RAM and Intel Xeon $2.3 \mathrm{GHz}$ processors took 191 minutes and 54 GB RAM. However, memory usage increases exponentially with more data points ( $\sim 100,000$ contigs $\approx 1$ TB RAM).

\section{Concluding Remarks}

Experimental testing on both real and artificial communities demonstrated that BinSanity+refinement outperformed the binning methods CONCOCT, MetaBat, MaxBin, MyCC, and GroopM when the coverage values for five or more metagenomic samples are available (In some cases BinSanity outperformed BinSanity+refinement). Below four metagenomes, composition information becomes more essential for BinSanity to correctly assign contigs. With this refinement step, BinSanity can maintain high precision and recall across a variety of community types. Based on the unsupervised binning of the infant gut and artificial communities, BinSanity (and BinSanity+refinement) consistently produces results with higher precision, completeness, and ARI compared to other unsupervised methods. Manually curated results generated similar outcomes, though the time spent manually refining bins can become a limiting factor as microbial community complexity increases. BinSanity had more success at consistently generating accurate genomes from strain- and species-level diversity. The consistency with which BinSanity generates high-quality genomes across varying community structures indicates that it is a good alternative to current methods for clustering of metagenomic data.

\section{Software Availability}

The most recent version of BinSanity is available at https://github.com/edgraham/BinSanity/

\section{References}

Alneberg J, Bjarnason BS, de Bruijn I, Schirmer M, Quick J, Ijaz UZ, Lahti L, Loman NJ, Andersson AF, and Quince C. 2014. Binning metagenomic contigs by coverage and composition. Nat Meth 11:11441146. 10.1038/nmeth.3103

Anantharaman K, Breier JA, and Dick GJ. 2016. Metagenomic resolution of microbial functions in deepsea hydrothermal plumes across the Eastern Lau Spreading Center. ISME J 10:225-239. 10.1038/ismej.2015.81 
410

411

412

413

414

415

416

417

418

419

420

421

422

423

424

425

426

427

428

429

430

431

432

433

434

435

436

437

438

439

440

441

442

443

444

445

446

447

448

449

450

451

452

453

454

455

456

Bohlin J, Snipen L, Hardy SP, Kristoffersen AB, Lagesen K, Dønsvik T, Skjerve E, and Ussery DW. 2010. Analysis of intra-genomic GC content homogeneity within prokaryotes. BMC Genomics 11:1-8. 10.1186/1471-2164-11-464

Bowers RM, Clum A, Tice H, Lim J, Singh K, Ciobanu D, Ngan CY, Cheng J-F, Tringe SG, and Woyke T. 2015. Impact of library preparation protocols and template quantity on the metagenomic reconstruction of a mock microbial community. BMC Genomics 16:1-12. 10.1186/s12864-0152063-6

Chen-Chia C, Yan-Cheng L, Jin-Tsong J, Chih-Kai C, and Zhi-Qian W. 2015. Feature genes selection of adult ALL microarray data with affinity propagation clustering. Consumer Electronics - Taiwan (ICCE-TW), 2015 IEEE International Conference on. p 230-231.

Chen SL, Lee W, Hottes AK, Shapiro L, and McAdams HH. 2004. Codon usage between genomes is constrained by genome-wide mutational processes. Proceedings of the National Academy of Sciences of the United States of America 101:3480-3485. 10.1073/pnas.0307827100

Dick GJ, Andersson AF, Baker BJ, Simmons SL, Thomas BC, Yelton AP, and Banfield JF. 2009. Communitywide analysis of microbial genome sequence signatures. Genome Biology 10:1-16. 10.1186/gb2009-10-8-r85

Eren AM, Esen ÖC, Quince C, Vineis JH, Morrison HG, Sogin ML, and Delmont TO. 2015. Anvi'o: an advanced analysis and visualization platform for 'omics data. PeerJ 3:e1319. 10.7717/peerj.1319

Flynn SD, and Moneypenny NF. 2013. Affinity propagation in adaptive network-based systems. Google Patents.

Frey BJ, and Dueck D. 2007. Clustering by Passing Messages Between Data Points. Science 315:972-976. 10.1126/science. 1136800

Fujiwara Y, Nakatsuji M, Shiokawa H, Ida Y, and Toyoda M. 2015. Adaptive Message Update for Fast Affinity Propagation. Proceedings of the 21th ACM SIGKDD International Conference on Knowledge Discovery and Data Mining. Sydney, NSW, Australia: ACM. p 309-318.

Gan G, and Ng MK-P. 2015. Subspace clustering using affinity propagation. Pattern Recognition 48:14551464.

Handelsman J, Rondon MR, Brady SF, Clardy J, and Goodman RM. 1998. Molecular biological access to the chemistry of unknown soil microbes: a new frontier for natural products. Chemistry \& Biology 5:R245-R249. 10.1016/S1074-5521(98)90108-9

Hassanabadi B, Shea C, Zhang L, and Valaee S. 2014. Clustering in Vehicular Ad Hoc Networks using Affinity Propagation. Ad Hoc Networks 13, Part B:535-548.

Hubert L, and Arabie P. 1985. Comparing partitions. Journal of Classification 2:193-218. 10.1007/bf01908075

Imelfort M, Parks D, Woodcroft BJ, Dennis P, Hugenholtz P, and Tyson GW. 2014. GroopM: an automated tool for the recovery of population genomes from related metagenomes. PeerJ 2:e603. 10.7717/peerj.603

J T Staley a, and Konopka A. 1985. Measurement of in Situ Activities of Nonphotosynthetic Microorganisms in Aquatic and Terrestrial Habitats. Annual Review of Microbiology 39:321-346. doi:10.1146/annurev.mi.39.100185.001541

Kanaya S, Yamada Y, Kudo Y, and Ikemura T. 1999. Studies of codon usage and tRNA genes of 18 unicellular organisms and quantification of Bacillus subtilis tRNAs: gene expression level and species-specific diversity of codon usage based on multivariate analysis. Gene 238:143-155.

Kang DD, Froula J, Egan R, and Wang Z. 2015. MetaBAT, an efficient tool for accurately reconstructing single genomes from complex microbial communities. PeerJ 3:e1165.

Langmead B, and Salzberg SL. 2012. Fast gapped-read alignment with Bowtie 2. Nature methods 9:357359. 10.1038/nmeth.1923 
457

458

459

460

461

462

463

464

465

466

467

468

469

470

471

472

473

474

475

476

477

478

479

480

481

482

483

484

485

486

487

488

489

490

491

492

493

494

495

496

497

498

499

500

501

502

503

504

Leone $M$, Sumedha, and Weigt M. 2007. Clustering by soft-constraint affinity propagation: applications to gene-expression data. Bioinformatics 23:2708-2715. 10.1093/bioinformatics/btm414

Li H, Handsaker B, Wysoker A, Fennell T, Ruan J, Homer N, Marth G, Abecasis G, Durbin R, and Genome Project Data Processing S. 2009. The Sequence Alignment/Map format and SAMtools. Bioinformatics 25:2078-2079. 10.1093/bioinformatics/btp352

Lin $\mathrm{H}-\mathrm{H}$, and Liao Y-C. 2016. Accurate binning of metagenomic contigs via automated clustering sequences using information of genomic signatures and marker genes. Scientific Reports 6:24175. 10.1038/srep24175

Lu YY, Chen T, Fuhrman JA, and Sun F. 2016. COCACOLA: binning metagenomic contigs using sequence COmposition, read CoverAge, CO-alignment and paired-end read LinkAge. Bioinformatics. 10.1093/bioinformatics/btw290

Markowitz VM, Chen I-MA, Palaniappan K, Chu K, Szeto E, Pillay M, Ratner A, Huang J, Woyke T, Huntemann M, Anderson I, Billis K, Varghese N, Mavromatis K, Pati A, Ivanova NN, and Kyrpides NC. 2014. IMG 4 version of the integrated microbial genomes comparative analysis system. Nucleic Acids Research 42:D560-D567. 10.1093/nar/gkt963

Mehmood R, and Bie R. 2015. Optimal Preference Detection Based on Golden Section and Genetic Algorithm for Affinity Propagation Clustering. Wireless Algorithms, Systems, and Applications: 10th International Conference, WASA 2015, Qufu, China, August 10-12, 2015, Proceedings: Springer. p 253.

Meyer JL, Jaekel U, Tully BJ, Glazer BT, Wheat CG, Lin H-T, Hsieh C-C, Cowen JP, Hulme SM, Girguis PR, and Huber JA. 2016. A distinct and active bacterial community in cold oxygenated fluids circulating beneath the western flank of the Mid-Atlantic ridge. Scientific Reports 6:22541. $10.1038 /$ srep22541

Parks DH, Imelfort M, Skennerton CT, Hugenholtz P, and Tyson GW. 2015. CheckM: assessing the quality of microbial genomes recovered from isolates, single cells, and metagenomes. Genome Research. 10.1101/gr.186072.114

Pedregosa F, Varoquaux G, Gramfort A, Michel V, Thirion B, Grisel O, Blondel M, Prettenhofer P, Weiss $\mathrm{R}$, and Dubourg V. 2011. Scikit-learn: Machine learning in Python. The Journal of Machine Learning Research 12:2825-2830.

Pride DT, Meinersmann RJ, Wassenaar TM, and Blaser MJ. 2003. Evolutionary Implications of Microbial Genome Tetranucleotide Frequency Biases. Genome Research 13:145-158. 10.1101/gr.335003

Pruitt KD, Tatusova T, and Maglott DR. 2007. NCBI reference sequences (RefSeq): a curated nonredundant sequence database of genomes, transcripts and proteins. Nucleic Acids Research 35:D61-D65. 10.1093/nar/gkl842

Quinlan AR, and Hall IM. 2010. BEDTools: a flexible suite of utilities for comparing genomic features. Bioinformatics 26:841-842. 10.1093/bioinformatics/btq033

Rosenberg A, and Hirschberg J. 2007. V-Measure: A Conditional Entropy-Based External Cluster Evaluation Measure. EMNLP-CoNLL. p 410-420.

Sandberg R, Winberg G, Bränden C-I, Kaske A, Ernberg I, and Cöster J. 2001. Capturing Whole-Genome Characteristics in Short Sequences Using a Naïve Bayesian Classifier. Genome Research 11:14041409. 10.1101/gr.186401

Santos JM, and Embrechts M. 2009. On the Use of the Adjusted Rand Index as a Metric for Evaluating Supervised Classification. In: Alippi C, Polycarpou M, Panayiotou C, and Ellinas G, eds. Artificial Neural Networks - ICANN 2009: 19th International Conference, Limassol, Cyprus, September 1417, 2009, Proceedings, Part II. Berlin, Heidelberg: Springer Berlin Heidelberg, 175-184.

Sharon I, Morowitz MJ, Thomas BC, Costello EK, Relman DA, and Banfield JF. 2013. Time series community genomics analysis reveals rapid shifts in bacterial species, strains, and phage during infant gut colonization. Genome Research 23:111-120. 10.1101/gr.142315.112 
505
Tully BJ, and Heidelberg JF. 2016. Potential Mechanisms for Microbial Energy Acquisition in Oxic DeepSea Sediments. Applied and Environmental Microbiology 82:4232-4243. 10.1128/aem.01023-16

Tully BJ, Sachdeva R, Heidelberg KB, and Heidelberg JF. 2014. Comparative genomics of planktonic Flavobacteriaceae from the Gulf of Maine using metagenomic data. Microbiome 2:34. 10.1186/2049-2618-2-34

Walter SF, Fischer B, and Buhmann JM. 2007. Clustering by affinity propagation. Master's thesis, Department of Physics, ETH Zurich, Switzerland. Procedia Computer Science 00 (2014) 1-9.

Wu Y-W, Tang Y-H, Tringe SG, Simmons BA, and Singer SW. 2014. MaxBin: an automated binning method to recover individual genomes from metagenomes using an expectation-maximization algorithm. Microbiome 2:1.

Zhengdong L, and Carreira-Perpinan MA. 2008. Constrained spectral clustering through affinity propagation. Computer Vision and Pattern Recognition, 2008 CVPR 2008 IEEE Conference on. p 1-8.

Zhou F, Olman V, and Xu Y. 2008. Barcodes for genomes and applications. BMC Bioinformatics 9:1-11. 10.1186/1471-2105-9-546 